\title{
Effects of monetary incentives on rehearsal and on cued recall
}

\author{
MICHAEL W. EYSENCK and M. CHRISTINE EYSENCK \\ Birkbeck College, University of London, London WCIE 7HX, England
}

\begin{abstract}
Words associated with high monetary incentives were better recalled than those associated with low incentives. It was found that high-incentive words were rehearsed more than lowincentive words and that the memorial advantage of high-incentive items was greater when the retrieval cues were weakly associated to the list words than when they were strong associates. It was concluded that the disproportionate amount of rehearsal given to high-incentive items led to greater elaboration or extensiveness of encoding, which in turn produced the observed memorial superiority of high-incentive items.
\end{abstract}

If subjects are asked to learn a list of words, half of which are associated with high monetary incentives and the remainder with low monetary incentives, the typical finding is that the high-incentive items are better remembered than the low-incentive items (e.g., Harley, 1965; Nelson, 1976). Atkinson and Wickens (1971) explained this finding by assuming that the probability of an item entering the limited-capacity rehearsal buffer and being rehearsed was greater for high-incentive items than for low-incentive items. Since they also assumed that long-term storage depended on the amount of rehearsal activity in the rehearsal buffer, it follows that highincentive material should be better remembered than low-incentive material.

The emphasis in the Atkinson and Wickens (1971) approach is primarily on possible quantitative effects of incentive on attention and rehearsal activity. However, it is entirely possible that incentive manipulations affect processing in a qualitative fashion as well as the purely quantitative. Indeed, the evidence suggests that more than one kind of rehearsal activity can be identified; for example, Craik and Lockhart (1972) proposed a distinction between maintenance rehearsal (i.e., mere repetition of processing activity) and elaboration rehearsal (i.e., rehearsal that extends the variety of stimulus processing).

This study was designed to clarify the nature of the enhanced memory performance produced by incentives. The role of rehearsal was investigated by means of the overt rehearsal technique (Rundus \& Atkinson, 1970), in which subjects are instructed to rehearse aloud any of the list items they choose during list presentation. An attempt was also made to investigate the effects of incentives on the nature and elaboration of processing by presenting different kinds of retrieval cues. The

Many thanks are due to the Social Science Research Council for their generous financial assistance during the preparation of this manuscript. Thanks are also due to Douglas Nelson for providing us with his unpublished associative norms. retrieval cues were either semantically or phonemically related to list words, and they were either strong or weak associates of list items. The general orienting assumptions were that qualitative effects of incentives on processing would be reflected in differential effects of incentives on semantic and phonemic retrieval, whereas quantitative effects of incentives on processing elaboration on retrieval to weak and strong cues.

\section{METHOD}

\section{Subjects}

There were 15 subjects. They were students at the University of London within the 18- to 30-year age range, and they received modest payment for their services.

\section{Materials and Design}

The subjects received four lists of words, each list consisting of 24 words. The four lists were presented in a number of different orders. All the words used had frequencies in excess of $50 /$ million according to the Thorndike and Lorge (1944) word count. Within each list, 12 words were high-incentive items and 12 were low-incentive items. The high-incentive words were printed in red capital letters, and the low-incentive words were printed in black capital letters; the two kinds of items were randomly distributed throughout each list. Subjects were offered 10 pence for each high-incentive item recalled, compared with 2 pence for the recall of each low-incentive item. Each list word was presented manually on a card at a rate of one item every $4 \mathrm{sec}$.

All of the subjects engaged in overt rehearsal during the presentation of two lists and silent processing during the presentation of the other two lists. On overt rehearsal lists, subjects were free to rehearse any list words they chose, but they were asked to rehearse out loud. A portable tape recorder was used to record their rehearsal patterns. Approximately half of the subjects engaged in overt rehearsal on the first and fourth lists, and the remaining subjects rehearsed overtly on the second and third lists.

Immediately after the presentation of each list, there was a cued recall retention list. The retrieval cues were read to the subject, and the subject was asked to write down any list word that seemed appropriate to each cue. Half the cues were semantically related to list words (e.g., "JUMP associated with "), and the other half were phonemically related to list words (e.g., "FEAST rhymes with_"). Half the retrieval cues were strongly associated with the list words (mean association 
value $=15.23$ according to association norms supplied by Nelson, Note 1), and the remaining cues were weakly associated with the list words (mean association value $=4.63$ ). There were thus four kinds of cue: strong semantic, weak semantic, strong phonemic, and weak phonemic. For each list, three high-incentive words and three low-incentive words were cued with each of the four varieties of retrieval cue.

The initial instructions informed the subjects that they would receive four lists of words and that cued recall of the words from each list would be required. The exact nature of the four kinds of retrieval cue was also explained.

\section{RESULTS}

\section{Rehearsal Data}

The number of overt rehearsals of high- and lowincentive items on each of the two lists involving overt rehearsal was obtained from the tape recordings, and the summary data are given in Table 1 . The data were analyzed by means of a two-factor analysis of variance based on incentive conditions (high vs. low) and list position (first vs. second). (All effects described as significant involve $p<.05$.) There were significantly more rehearsals of high-incentive items than of lowincentive items $[\mathrm{F}(1,14)=9.57, \mathrm{MSe}=85.1]$. However, this main effect was qualified by a significant interaction between incentive conditions and list position; in this interaction, the superiority of the high-incentive items was more pronounced on the second list on which overt rehearsal occurred than on the first such list. Within the context of the Atkinson and Wickens (1971) theory, it may be that subjects learn the value of rehearsing important items more than unimportant items during the course of the experiment.

Table 1

Mean Overt Rehearsals as a Function of List Position and Incentive Conditions

\begin{tabular}{|c|c|c|c|c|}
\hline \multirow[b]{3}{*}{ Incentive } & \multicolumn{4}{|c|}{ List Position } \\
\hline & \multicolumn{2}{|c|}{ First } & \multicolumn{2}{|c|}{ Second } \\
\hline & Mean & SD & Mean & SD \\
\hline High & 22.33 & 11.51 & 26.53 & 15.01 \\
\hline Low & 17.93 & 10.27 & 16.20 & 7.61 \\
\hline
\end{tabular}

\section{Cued Recall Data}

Preliminary analyses of the cued recall data indicated that performance was unaffected by the presence or absence of overt rehearsal during presentation of the list on the study trial. Accordingly, the summary data for cued recall given in Table 2 are pooled across this variable. The cued recall data were analyzed by means of an analysis of variance comprising the four factors of list position, incentive, cue strength, and cue type (semantic vs. phonemic). All factors were within subjects. Recall was higher with strong retrieval cues than with weak retrieval cues $[\mathrm{F}(1,14)=112.53, \mathrm{MSe}=.58]$ and higher with phonemic cues than with semantic cues $[F(1,14)=27.96, \mathrm{MSe}=.76]$. In addition, recall was higher for high-incentive items than for low-incentive items $[\mathrm{F}(1,14)=19.25, \mathrm{MSe}=.89]$.

The above main effects must be seen in light of the significant interaction between incentive and cue strength $[\mathrm{F}(1,14)=22.52, \mathrm{MSe}=.44]$. Analysis of the simple main effects indicated that there was a substantial effect of incentives with weak retrieval cues $[F(1,28)=$ $40.75, \mathrm{MSe}=.57 \mathrm{]}$, but there was no effect of incentives with strong retrieval cues $[F(1,28)=.26]$.

\section{DISCUSSION}

The expectation from the theoretical position of Atkinson and Wickens (1971) was that high-incentive items would be attended to and rehearsed to a greater extent than low-incentive items. This expectation was supported by the finding that highincentive items received significantly more overt rehearsals than did low-incentive items. Since high-incentive items were also better remembered than low-incentive items, it is tempting to suppose that the effect of incentives on memory was mediated by differential rehearsal, in spite of the fact that this is an argument of the post hoc ergo propter hoc variety.

There are at least two possible interpretations of the finding that high-incentive items showed a considerable memorial superiority over low-incentive items. On the one hand, subjects might engage in more extensive or elaborate encoding of highincentive items than of low-incentive items without altering the kind of processing employed. There is reasonable evidence that elaboration of encoding is positively related to memory performance (e.g., Klein \& Saltz, 1976). On the other hand, incentives might lead subjects to change their processing strategy, with the result that high-and low-incentive items would manifest qualitative differences in encoding. For example, if high-incentive items tended to be processed semantically, whereas low-incentive

Table 2

Cued Recall as a Function of Cue Type, Incentive Conditions, and List Position

Cue Type

\begin{tabular}{|c|c|c|c|c|c|c|c|c|c|c|c|c|c|c|c|c|}
\hline \multirow[b]{3}{*}{ List } & \multicolumn{8}{|c|}{ High-Incentive Condition } & \multicolumn{8}{|c|}{ Low-Incentive Condition } \\
\hline & \multicolumn{2}{|c|}{$\begin{array}{c}\text { Strong } \\
\text { Semantic }\end{array}$} & \multicolumn{2}{|c|}{$\begin{array}{c}\text { Strong } \\
\text { Phonemic }\end{array}$} & \multicolumn{2}{|c|}{$\begin{array}{c}\text { Weak } \\
\text { Semantic }\end{array}$} & \multicolumn{2}{|c|}{$\begin{array}{c}\text { Weak } \\
\text { Phonemic }\end{array}$} & \multicolumn{2}{|c|}{$\begin{array}{c}\text { Strong } \\
\text { Semantic }\end{array}$} & \multicolumn{2}{|c|}{$\begin{array}{c}\text { Strong } \\
\text { Phonemic } \\
\end{array}$} & \multicolumn{2}{|c|}{$\begin{array}{c}\text { Weak } \\
\text { Semantic }\end{array}$} & \multicolumn{2}{|c|}{$\begin{array}{c}\text { Weak } \\
\text { Phonemic }\end{array}$} \\
\hline & Mean & SD & Mean & SD & Mean & SD & Mean & $\mathrm{SD}$ & Mean & SD & Mean & SD & Mean & SD & Mean & SD \\
\hline 1 & 1.7 & .8 & & .68 & & .8 & & & & .96 & 1.93 & .88 & .60 & 0 & 1.27 & 1.03 \\
\hline 2 & & 1 & & .7 & & .7 & & & & & & & 4 & & 1.13 & .83 \\
\hline 3 & 1.3 & $1: 18$ & & .64 & & .8 & 1.4 & 1.0 & & .9 & 1. & & .4 & .6 & & .88 \\
\hline 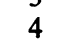 & 1.60 & .83 & 2.13 & .74 & 1.00 & .76 & 1.73 & .80 & 2.00 & .84 & 1.87 & .74 & .27 & .80 & .73 & .70 \\
\hline
\end{tabular}


items were processed phonemically, this would typically produce a large effect of incentive magnitude on memory (cf. Craik \& Lockhart, 1972).

If a major effect of incentives were to increase the probability that the stimulus material would be processed semantically, then an interaction between incentive conditions and cue type (semantic vs. phonemic) would be predicted, in which incentive effects on recall would be greater with semantic than with phonemic cues. In fact, the incentive effect was comparable for both semantic and phonemic cues, thus suggesting that incentives did not produce qualitative changes in processing. It may be of relevance that naive subjects show little awareness of the fact that the kind of processing performed on presented stimuli can affect dramatically their subsequent memory for such stimuli (Cutting, 1975); there is no particular reason why unaware subjects would change their processing strategies.

While there was little evidence that incentives altered processing strategies, there was support for the hypothesis that highincentive stimuli are processed more extensively or elaborately than low-incentive stimuli. If low-incentive words were primarily processed in terms of the most obvious and readily accessible features, whereas high-incentive words were processed in terms of both readily accessible and less accessible features, then it would be reasonable to assume that the incentive manipulation would interact with cue strength. In such an interaction, the enhancing effect of high incentives on cued recall would be greater when weak retrieval cues were used than when strong retrieval cues were used. In fact, the data were entirely consistent with this prediction, since high-incentive words were much better recalled than low-incentive words to weak retrieval cues, but there was no incentive effect at all with strong retrieval cues.

In summary, the various findings of this study suggest that there may be a relatively straightforward explanation of the enhancing effect of high incentives on retrieval. Subjects spend a disproportionate amount of the available processing time attending to and rehearsing high-incentive stimuli, and the effect of this additional rehearsal is to increase the extensiveness or elaboration of encoding by enlarging the number of encoded features or attributes. This increase in the elaboration of encod- ing will facilitate memory performance in a wide variety of retrieval environments. Finally, while incentives affect the extensiveness of processing, they do not directly affect the "depth" or semanticity of processing.

\section{REFERENCE NOTE}

1. Nelson, D. L. Personal communication, March 1976.

\section{REFERENCES}

Atrinson, R. C., \& Wickens, T. D. Human memory and the concept of reinforcement. In R. Glaser (Ed.), The nature of reinforcement. London: Academic Press, 1971.

Craik, F. I. M., \& Lockhart, R. S. Levels of processing: A framework for memory research. Journal of Verbal Learning and Verbal Behavior, 1972, 11, 671-684.

Cutting, J. E. Orienting tasks affect recall performance more than subjective impressions of ability to recall. Psychological Reports, 1975, 36, 155-158.

HarLEY, W. F. The effect of monetary incentive in paired associate learning using a differential method. Psychonomic Science, 1965, 2, 377-378.

KLEIN, K., \& SALTZ, E. Specifying the mechanisms in a levels-ofprocessing approach to memory. Journal of Experimental Psychology: Human Learning and Memory, 1976, 2, 671-679.

NeLSON, T. O. Reinforcement and human memory. In W. K. Estes (Ed.), Handbook of learning and cognitive processes (Vol. 3). Hillsdale, N.J: Erlbaum, 1976.

Rundus, D., \& Atrinson, R. C. Rehearsal processes in free recall, a procedure for direct observation. Journal of Verbal Learning and Verbal Behavior, 1970, 9, 99-105.

ThorNdike, E. L., \& LoRge, I. The teacher's wordbook of 30,000 words. New York: Columbia University, Teachers College, Bureau of Publications, 1944.

(Received for publication January 16, 1980.) 